\title{
Defective podocyte XBP1 signalling in diabetic nephropathy
}

Endoplasmic reticulum (ER) dysfunction has been linked to diabetic nephropathy (DN); however, the mechanisms underlying dysfunctional ER signalling in DN are unclear. Thati Madhusudhan and colleagues now report that defective insulin signalling in podocytes induces a maladaptive ER-stress response by disrupting p85-mediated nuclear translocation and activation of XBP1 - a key regulator of the unfolded protein response (UPR) within the ER.

The UPR functions to restore ER homeostasis by folding or degrading misfolded proteins. One branch of the

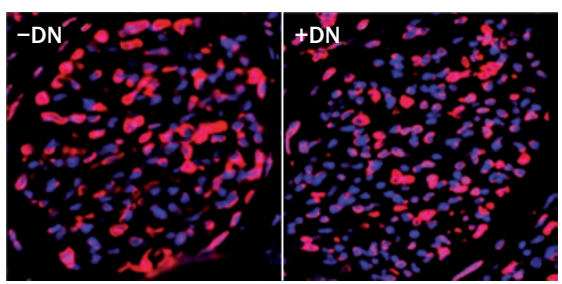

Nuclear localization of XBP1 in biopsy samples from patients without (-DN) and with (+DN) DN. Permission obtained from Nature Publishing Group @ $\odot$ Madhusudhan, T. et al. Nat. Commun. 6, 6496 (2015).
UPR is regulated by the transcription factor XBP1. In mouse models of DN, Madhusudhan and colleagues found that nuclear translocation of XBP1 was impaired, and associated with increased levels of the transcription factors ATF6 and CHOP. They also found that nuclear localization of XBP1 was reduced in renal biospy samples from humans with DN.

To determine whether hyperglycaemia induces ER stress, the researchers treated diabetic mice with either an SGLT2 inhibitor or a chemical chaperone to attenuate ER stress. Both treatments normalized nuclear levels of XBP1, ATF6 and CHOP and attenuated DN. Conversely, addition of glucose to cultured podocytes and glomerular epithelial cells impaired nuclear translocation of XBP1 and increased levels of ATF6 and CHOP.

The researchers then used genetic models to assess the pathophysiologic role of XBP1 and ATF6 in DN. Podocytespecific deletion of XBP1 or induction of ATF6 exacerbated indices of DN. To investigate the role of dysregulated insulin signalling in this process, the researchers generated mice with podocyte-specific deletion of the insulin receptor. Mice with defective insulin signalling in podocytes showed impaired nuclear translocation of XBP1 and exacerbated features of $\mathrm{DN}$. Moreover, genetic inactivation of the downstream regulators of insulinreceptor signalling - p85 $\alpha$ and p85 $\beta$ impaired XBP1 nuclear translocation and exacerbated DN.

"These results shed new light onto the regulation of the UPR in DN and identify a new function of insulin signalling in podocytes," state the researchers. "We hope future studies will identify pathways to rescue defective insulin signalling in podocytes and evaluate whether such rescue is sufficient to provide cytoprotection to podocytes in DN."

Susan J. Allison

Original article Madhusudhan, T. et al. Defective podocyte insulin signalling through p85-XBP1 promotes ATF6dependent maladaptive ER-stress response in diabetic nephropathy. Nat. Commun. 6, 6496 (2015) 